\title{
Theoretical Support for the Hydrodynamic Mechanism of Pulsar Kicks
}

\author{
J. Nordhaus, ${ }^{1,}$, T. D. Brandt,${ }^{1}$ A. Burrows, ${ }^{1}$ E. Livne, ${ }^{2}$ and C. D. $\mathrm{Ott}^{3}$ \\ ${ }^{1}$ Department of Astrophysical Sciences, Princeton University, Princeton, NJ 08544, U.S.A. \\ ${ }^{2}$ Racah Institute of Physics, Hebrew University, Jerusalem, Israel \\ ${ }^{3}$ Theoretical Astrophysics, Mail Code 350-17, California Institute of Technology, Pasadena, CA 91125 U.S.A.
}

(Dated: October 29, 2018)

\begin{abstract}
The collapse of a massive star's core, followed by a neutrino-driven, asymmetric supernova explosion, can naturally lead to pulsar recoils and neutron star kicks. Here, we present a two-dimensional, radiation-hydrodynamic simulation in which core collapse leads to significant acceleration of a fullyformed, nascent neutron star (NS) via an induced, neutrino-driven explosion. During the explosion, a $\sim 10 \%$ anisotropy in the low-mass, high-velocity ejecta lead to recoil of the high-mass neutron star. At the end of our simulation, the NS has achieved a velocity of $\sim 150 \mathrm{~km} \mathrm{~s}^{-1}$ and is accelerating at $\sim 350 \mathrm{~km} \mathrm{~s}^{-2}$, but has yet to reach the ballistic regime. The recoil is due almost entirely to hydrodynamical processes, with anisotropic neutrino emission contributing less than $2 \%$ to the overall kick magnitude. Since the observed distribution of neutron star kick velocities peaks at $\sim 300-400$ $\mathrm{km} \mathrm{s}^{-1}$, recoil due to anisotropic core-collapse supernovae provides a natural, non-exotic mechanism with which to obtain neutron star kicks.
\end{abstract}

PACS numbers: 97.60.Bw, 97.60.Gb, 97.60.Jd, 95.30.Jx, 95.30.Lz

\section{INTRODUCTION}

The velocity distribution of young pulsars bears little resemblance to that of their massive star progenitors 1. Typical birth velocities range from $\sim 200-500$ $\mathrm{kms}^{-1}$, with some reaching upwards of $\sim 1000 \mathrm{~km} \mathrm{~s}^{-1}$ [2]. While the observed pulsar velocities may hint at a two-component distribution (possibly implying two populations) 3 5], recent work supports a single, Maxwellian distribution [6] 10 .

Various mechanisms for the origin of neutron star kicks and pulsar recoil and their connections with pulsar spins have been proposed 11. Misaligned jet/counter-jets during the supernova explosion might produce sufficient acceleration if they are launched near the proto-neutron star (PNS) [12, 13]. However, such jets are generated only in fast rotators and may not be generic [14 16]. Another possibility is anisotropic neutrino emission from the cooling proto-neutron star. If strong magnetic fields are present, neutrino-matter interactions can generate dipole asymmetries of $\sim 1 \%$, leading to recoil on the order of a few hundred $\mathrm{kms}^{-1}[17 \sqrt[20]{2}$. These scenarios require magnetar field strengths (i.e. $10^{14}-10^{15} \mathrm{G}$ ) and/or exotic neutrino physics [21 24] and may not produce substantial kicks in typical core-collapse supernovae.

If neutron star kicks are a generic feature of core collapse, then the most natural explanation is recoil due to an asymmetric supernova explosion [25 28]. During axisymmetric core collapse, the stalled bounce shock is unstable to neutrino-driven convection and low-order $\ell$ modes. Significant asymmetry at the onset of neutrino-

*Electronic address: nordhaus@astro.princeton.edu; tbrandt@astro.princeton.edu; burrows@astro.princeton.edu; livne@phys.huji.ac.il; cott@tapir.caltech.edu driven shock revival should naturally lead to an asymmetric explosion and the hydrodynamic recoil of the PNS [25, 26, 28, 30].

Observations of large-scale asymmetries in young supernova remnants lend qualitative support to the hydrodynamic mechanism 32. Unfortunately, multidimensional, radiation-hydrodynamic simulations of recoil are computationally challenging. A proper study requires simulating the full physics of collapse, the formation of the PNS, the development of instabilities during the post-bounce phase, the evolution of the asymmetric explosion, the off-axis movement of PNS, and the full decoupling of the ejecta from the PNS. Because the expanding post-shock material interacts with the PNS through both pressure and gravity, this requires following the shock out to large distances (hundreds of thousands of kilometers) and late times (several seconds). Complicating matters is that during this evolution, one must continue to resolve the movement of the PNS and the surrounding highly nonlinear flow.

Scheck et al. 2006 present a practical approach to this problem [26, 28]. By excising the PNS and replacing it with a rigid, contracting boundary, they avoid severe Courant timestep restrictions. They also greatly simplify their radiation transport, enforcing a constant luminosity at their inner boundary, and begin their calculations 20 ms after bounce. These approximations allow Scheck et al. to follow the evolution of the shock to large distances and late times, and to perform a detailed parameter study. Unfortunately, this approach requires them to infer a kick through a rigid, impenetrable boundary. Their results should therefore be checked by more realistic (though costly) simulations.

As a complement to the work of Scheck et al., we present a two-dimensional (2D) simulation of the collapse of a $15-M_{\odot}$ progenitor core. By employing a pseudoCartesian mesh at the center of our domain, we naturally 
capture the neutron star's formation and any subsequent off-center acceleration. During our simulation, the protoneutron star forms, after which it recoils due to a delayed, neutrino-driven, anisotropic explosion. The explosion is artificially induced by adding additional neutrino luminosity to the calculation. At the end of our simulation, the NS has achieved a velocity of $\sim 150 \mathrm{~km} \mathrm{~s}^{-1}$ and is still accelerating at $\sim 350 \mathrm{~km} \mathrm{~s}^{-2}$. The recoil is primarily hydrodynamic in nature, with anisotropic neutrino emission contributing less than $2 \%$ of the overall kick magnitude. Most notably, we obtain a significant kick without invoking strong magnetic fields, exotic neutrino physics, or misaligned jets. Our results are consistent with the previous Scheck et al. studies [26, 28]. Taken together, these simulations provide compelling numerical support for the hydrodynamic mechanism of neutron star kicks.

\section{NUMERICAL SETUP AND METHODS}

Our 2D, axisymmetric calculations are performed with the multi-group, arbitrary Lagrangian-Eulerian (ALE), radiation-hydrodynamics code VULCAN/2D [33]. We perform $2 \mathrm{D}$ radiation transport using the multi-group flux-limited diffusion approximation [34]. We simulate the collapse of the inner $5000 \mathrm{~km}$ of a non-rotating, $15-M_{\odot}$, solar-metallicity, red-supergiant progenitor 35. Exterior to $20 \mathrm{~km}$, our computational domain is a spherical-polar mesh which transitions to a pseudoCartesian grid in the center. Such a grid avoids severe timestep restrictions due to the convergence of angular zones and frees the PNS to move in response to radiation or hydrodynamic forces. Our mesh covers the full $180^{\circ}, 2 \mathrm{D}$ domain with 120 angular zones and 330 radial zones (logarithmically spaced exterior to the inner Cartesian region). We employ the finite-temperature nuclear equation of state of Shen et al. [36, 37] and include selfgravity with a grid-based solution of the Poisson equation [38. To ensure that we optimally resolve the high-density core, we allow our grid to track the PNS. Our remapping scheme determines the center of mass of the inner core (i.e. densities above $10^{12} \mathrm{~g} \mathrm{~cm}^{-3}$ ) after each timestep and shifts the mesh to keep the core centered while ensuring momentum conservation.

Despite decades of intense theoretical effort, the success of the delayed-neutrino mechanism [39 41] in driving core-collapse supernova explosions has still not been demonstrated [31, 42,51]. However, recent calculations have shown that this mechanism's capacity to power explosions increases with dimension [52, 53]. Ambitious three-dimensional calculations with accurate neutrino transport may yet validate the delayed-neutrino mechanism.

Because previous core-collapse studies with VUL$\mathrm{CAN} / 2 \mathrm{D}$ did not produce neutrino-driven supernovae [14, 27, 54, 56, we induce explosions by supplementing the radiation transport with additional electron and antielectron neutrino luminosity $\left(L_{\nu_{e}}=L_{\bar{\nu}_{e}}=2 \times 10^{52}\right.$
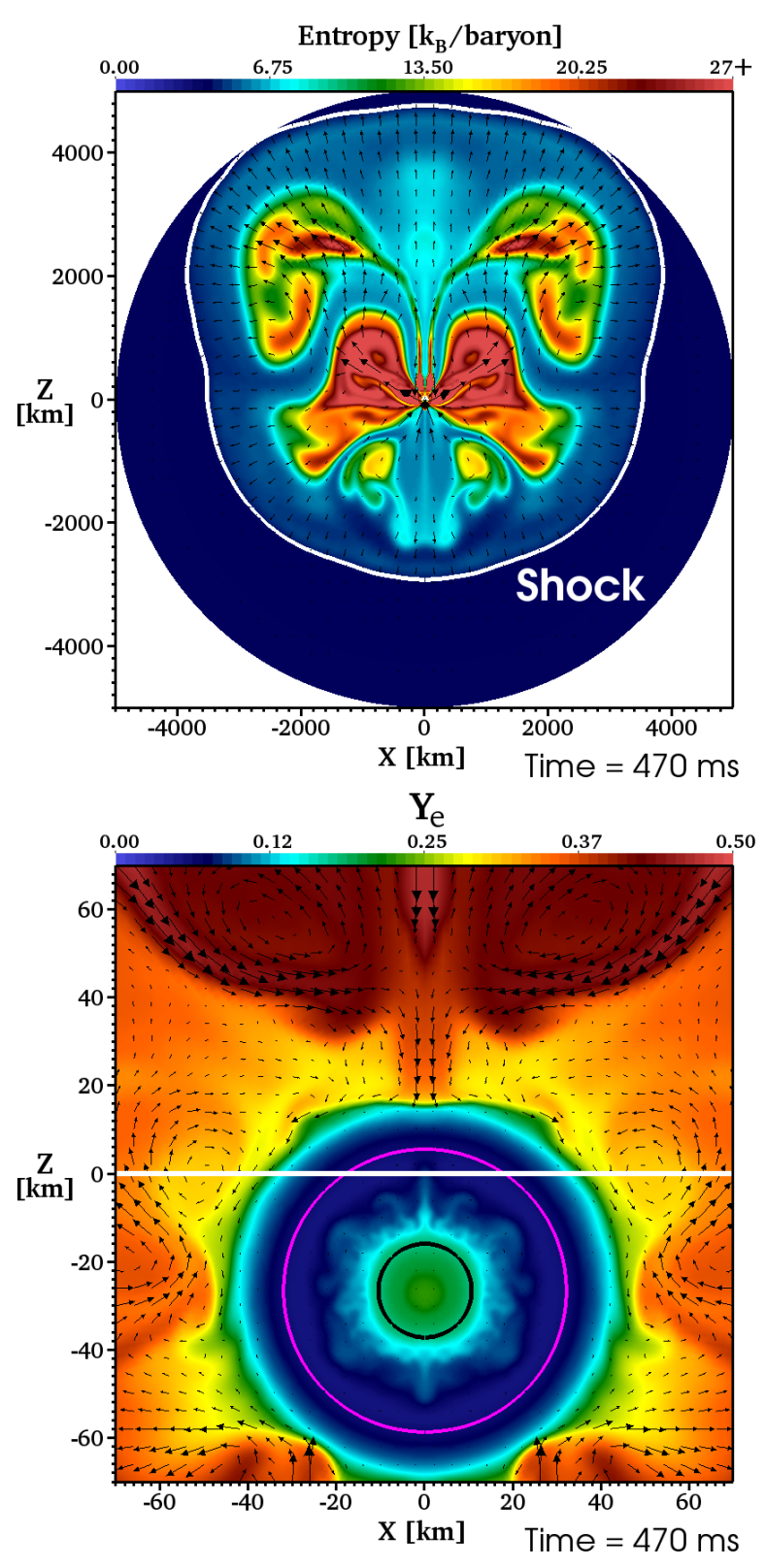

FIG. 1: The recoil of the proto-neutron star due to an asymmetric core-collapse supernova explosion. The large-scale explosion is primarily in the $+Z$ direction (top) while the PNS is kicked in the $-Z$ direction (bottom). In the bottom panel, the white line denotes $Z=0$, while the purple and black curves represent the isodensity surfaces where $\rho=10^{12}$ and $10^{14} \mathrm{~g} \mathrm{~cm}^{-3}$, respectively. Velocity vectors are overlaid in black.

$\operatorname{erg~s}^{-1}$ ) as described in [52, 53]. This represents an enhancement in the $\nu_{e}$ and $\bar{\nu}_{e}$ luminosities of $\sim 50 \%$. The core collapses to nuclear densities, launching a bounce shock which stalls and is subsequently revived mainly by charged-current neutrino absorption after a delay of approximately 135 milliseconds. 


\section{RECOIL FROM ASYMMETRIC CORE-COLLAPSE EXPLOSIONS}

At the onset of explosion, the hydrodynamic flow behind the shock is turbulent and the shock itself is deformed by the development of low-mode instabilities 26, 28, 47, 57, 58. The PNS recoils due to the blast's anisotropic propagation through the stellar envelope. We follow the explosion and the acceleration of the PNS until $470 \mathrm{~ms}$ after bounce, at which point the shock front reaches the boundary of our computational domain $(5000$ $\mathrm{km})$. Figure 1 shows the global explosion geometry and the position of the PNS at the end of our calculation. The top panel is an entropy map of our computational domain with velocity vectors overlaid and the shock position outlined in white. The bottom panel shows the electron fraction $Y_{\mathrm{e}}$ over the inner $\sim 70 \mathrm{~km}$. The white line is the $Z=0$ axis, while the pink and black curves represent the $10^{12} \mathrm{~g} \mathrm{~cm}^{-3}$ and $10^{14} \mathrm{~g} \mathrm{~cm}^{-3}$ isodensity contours, respectively. Note that the asymmetry of the explosion in the $+Z$-direction leads to a PNS recoil in the $-Z$-direction. While axisymmetry restricts our core to motion along the $Z$-axis, three-dimensional computations would impose no such constraint and could produce a recoil in any direction for initially non-rotating progenitors. Note that the presence of rotation may lead to a preferred explosion direction and, hence, kick direction. The differences between kicks from non-rotating and rotating progenitor models should be investigated in 3D.

While VULCAN/2D automatically and selfconsistently computes the acceleration of the core, it does not output the individual forces governing the motion of the PNS. We therefore post-process our results by computing the hydrodynamic acceleration $\vec{a}_{\mathrm{c}}$ of the core due to anisotropic gravitational forces, pressure forces, and momentum flux. The Eulerian equations of hydrodynamics give

$\vec{a}_{\mathrm{c}}=\dot{\vec{v}}_{\mathrm{c}} \sim \int_{r>r_{\mathrm{c}}} \frac{G \vec{r}}{r^{3}} d m-\frac{1}{M_{\mathrm{c}}}\left[\oint_{r=r_{\mathrm{c}}} P d \vec{S}+\oint_{r=r_{\mathrm{c}}} \rho v_{\mathrm{r}} \vec{v} d S\right]$

where $\rho$ is the density, $M_{\mathrm{c}}$ and $\vec{v}_{\mathrm{c}}$ are the mass and mean velocity of the inner region (where $\rho \geq 10^{12} \mathrm{~g} \mathrm{~cm}^{-1}$ ), $P$ is the gas pressure, $\vec{v}$ is the fluid velocity, $v_{\mathrm{r}}$ is the radial component of the velocity, and $r_{\mathrm{c}}$ is a fiducial spherical radius. The code self-consistently yields the recoil speed of the PNS (approximately bounded by the purple curve in Fig. 1), but we can use Eq. 1 to determine the various contributions to its acceleration and consequent motion.

The first term in Eq. 1 represents the acceleration due to the gravitational field exterior to $r_{\mathrm{c}}$, assuming a spherically symmetric distribution of matter interior to this radius. The second term is due to anisotropic gas pressure, while the third term represents the contribution due to momentum flux. In a spherically symmetric explosion, each term would vanish individually. These three terms include all hydrodynamic forces, but do not include asymmetries in the radiation pressure. In our sim-
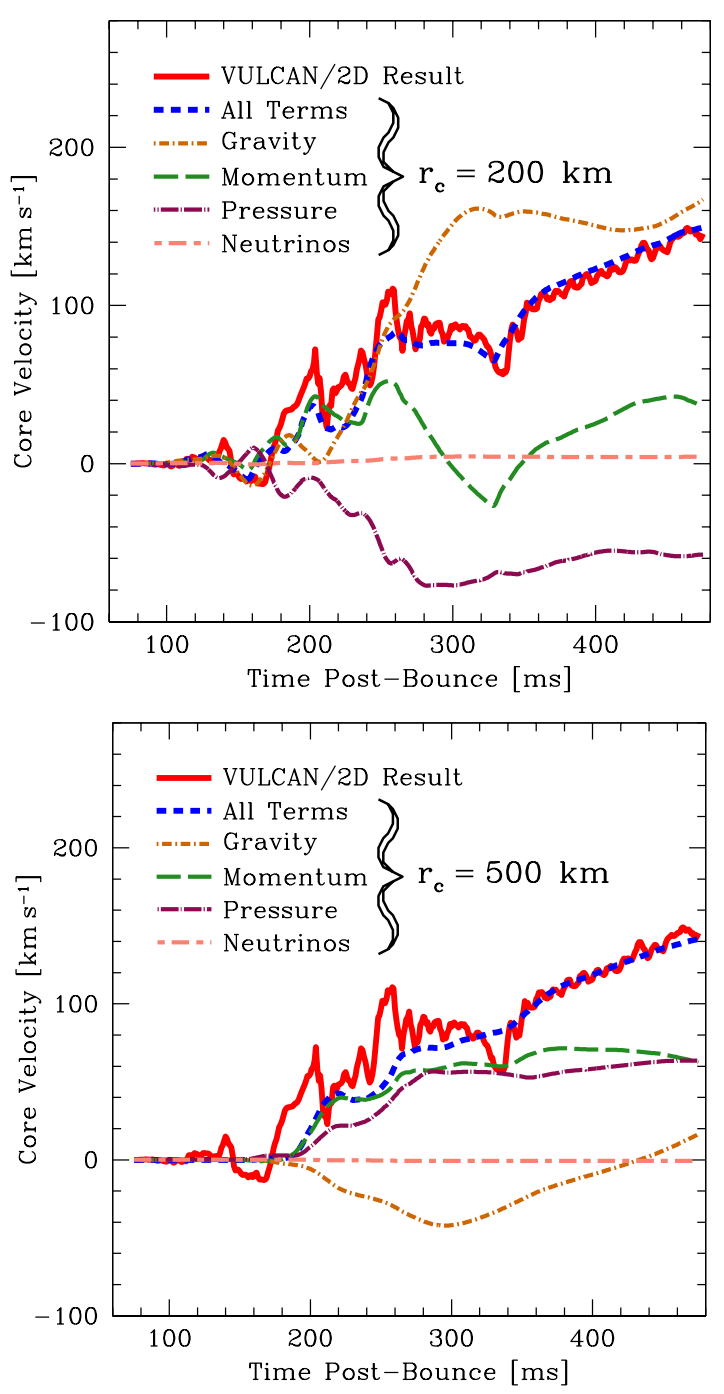

FIG. 2: The core velocity as a function of time after bounce. The solid-red curve in both figures shows the core velocity, in the $-Z$ direction, as a function of time after bounce in our simulation. Though the inferred core velocities calculated at $r_{\mathrm{c}}=200 \mathrm{~km}$ (top figure) and $r_{\mathrm{c}}=500 \mathrm{~km}$ (bottom figure) accurately reproduce the actual core velocity at late times, this figure demonstrates that one must exercise caution when interpreting the relative contribution of each component. Anisotropic neutrino flux contributes very little $(\lesssim 2 \%)$ of the total kick at all radii.

ulations, exterior to the radius at which the flux limiter transitions to free-streaming, anisotropic neutrino momentum contributes $\sim 2 \%$ of the total kick (see Fig. 2).

In general, the relative contributions of the various terms in Eq. 1 will depend sensitively on the radiationhydrodynamics and explosion dynamics. For instance, a spherically-symmetric distribution of ejected mass possessing asymmetric ejection velocities will lead to gravity and momentum terms of the same sign. In particular, since the PNS recoils towards the lower-velocity ejecta, 
the gravitational acceleration is in the same direction as the kick. This gravitational "tug-boat" effect enhances the recoil. Isotropic ejection velocities with anisotropic mass loss results in the gravity component partially canceling the momentum contribution.

We present the PNS kick velocity (as computed by VULCAN/2D) as a solid red line in both the top and bottom panels of Fig. 2 Using Eq. 1. we show the inferred kick velocity (dashed-blue curve) and its components at $200 \mathrm{~km}$ (top panel) and $500 \mathrm{~km}$ (bottom panel). These curves represent the mean velocities of matter interior to $200 \mathrm{~km}$ and $500 \mathrm{~km}$. As the core evolves, matter interior to $500 \mathrm{~km}$ becomes more centrally concentrated and its average velocity approaches that of the innermost regions (i.e. the monopole approximation gets better and better). The agreement between the red line and the blue lines therefore improves with time.

Figure 2 demonstrates that the kick imparted to the PNS may be inferred by evaluating Eq. 1 even at large radii. However, the relative contributions of the three terms in Eq. 1 differ dramatically. At $r_{\mathrm{c}}=200 \mathrm{~km}$, the late time evolution of our simulation is dominated by the gravitational component, while the momentum and pressure contributions are of opposite sign and comparable in magnitude. For $r_{\mathrm{c}}=500 \mathrm{~km}$, the pressure and momentum contributions are approximately equal (in both sign and magnitude) and nearly constant between $\sim 200 \mathrm{~ms}$ and $\sim 470 \mathrm{~ms}$. The secular evolution of the PNS velocity at the end of our calculation is governed by the gravitational component. The one component which does not depend strongly on radius is the contribution from anisotropic neutrino emission, which is small $(\lesssim 2 \%$ of the kick).

The interpretation of the kick (though not its value) thus depends on the radius at which the terms of Eq.11]are evaluated. At large radii, pressure and gravity vanish and an observer will attribute the entire kick to anisotropic momentum flux. The story is very different near the PNS itself. Because the inner core is nearly in hydrostatic equilibrium, pressure and gravity are both very large and in balance. An observer in this region would remark on the near cancellation of the gravitational and pressure terms in Eq. 1. For example, in our calculations, with a radius $r_{\mathrm{c}}$ that moves inward to always enclose $1.3 M_{\odot}$, these two components of the kick cancel to one part in $10^{2}$. Our results demonstrate the limitations of interpreting the individual components of Eq. 1. Since pressure and gravity do work on expanding matter, their contributions to the acceleration decrease in magnitude relative to the contribution due to the anisotropic momentum flux.

\section{A. Extrapolating the Kick}

Figure 2 indicates that our PNS is still accelerating at $\sim 350 \mathrm{~km} \mathrm{~s}^{-2}$ when the shock has reached the boundary of our computational domain. However, the ejecta have not yet decoupled from the core to reach the ballistic regime. The spatial distributions of momentum and velocity offer a hint of the core's future evolution, but unfortunately do not permit a straightforward extrapolation. Ideally (though at considerable computational expense), this would be handled by remapping our results onto a larger grid and continuing a full radiationhydrodynamic calculation. However, momentum and velocity maps, which we show in Fig. 3 , offer a useful picture of the ejecta at the end of our calculation.

The top panel of Fig. 3 shows the velocity of matter throughout our computational domain in units of the local escape speed, calculated assuming a spherically symmetric distribution of matter. Because the potential is dominated by the PNS, this approximation is extremely accurate. The map clearly shows that our model has not yet reached the ballistic regime, and that the matter behind the shock is still accelerating and evolving dynamically. A significant region of matter at $Z \sim-1000$ $\mathrm{km}$ seems likely to fall back, while a pocket of material at $Z \sim 2500 \mathrm{~km}$ is expanding at nearly twice the local escape speed. The infalling region has only $\sim 20 \%$ of the momentum in the core and, thus, is unlikely to significantly affect our inferred kick. However, the complexity of the hydrodynamics makes it impossible to extrapolate by assuming, for example, self-similar expansion.

The lower panel of Fig. 3 shows the projected $Z$ momentum density, $p_{Z} \equiv \pi R \rho v_{Z}$. The factor $\pi R$, where $R$ is the cylindrical radius, is the length of a semicircle of revolution. This projects the half-cylinder defined by $0<\phi<\pi$ in 3D onto the half-plane $X>0$ in 2D, so that $\int p_{Z} \mathrm{~d} X \mathrm{~d} Z$ gives the correct value for the total $Z$-momentum. This map shows that the high-velocity bubbles at $Z \sim 2500 \mathrm{~km}$ are regions of low density; most of the momentum is concentrated behind the shock and in the regions behind the highest velocity ejecta at $Z \sim 1000 \mathrm{~km}$. At the end of our calculation the PNS is still injecting mass and momentum into these regions. There appears to be no such injection of momentum into the regions at negative $Z$. If this causes the expansion of matter to slow in the $-Z$ direction, it could help maintain an asymmetric matter distribution, and thus the gravitational component of its acceleration, for several seconds.

The continued acceleration of the PNS will depend on the evolution of the asymmetry of shocked material. There are a variety of ways to quantify this asymmetry, as discussed in 28, 38. We choose $\alpha \equiv\left\langle v_{z}\right\rangle /\langle|v|\rangle$, where \langle\rangle denotes a mass-weighted average over the post-shock region with $r>100 \mathrm{~km}$ (to exclude the PNS itself). This is similar to the $\alpha$ presented in [28. If we assume this asymmetry to be constant in time, material on one side of the PNS will be a factor of $1-\alpha$ as close as material on the other side. We may then crudely estimate the gravitational acceleration of the core, $a_{c, \text { grav }}$ as

$$
a_{c, \text { grav }} \sim G M_{\mathrm{sh}}\left(\frac{1}{\left[(1-\alpha) r_{\mathrm{sh}}\right]^{2}}-\frac{1}{r_{\mathrm{sh}}^{2}}\right) \approx \frac{2 \alpha G M_{\mathrm{sh}}}{r_{\mathrm{sh}}^{2}}
$$

for small $\alpha$, where $r_{\mathrm{sh}}$ is the shock radius and $M_{\mathrm{sh}}$ is 
the total mass of ejecta and shocked envelope material. In our calculation, $\alpha \sim 0.1$ from 300 milliseconds to 470 milliseconds after bounce. Assuming $M_{\mathrm{sh}} \sim M_{\odot}$ and $\alpha \sim 0.1$, then for $a_{c, \text { grav }}$ to be of order $1 \mathrm{~km} \mathrm{~s}^{-2}$, we need to follow the shock out to $\sim 10^{5} \mathrm{~km}$. This corresponds to 5 seconds at a shock velocity of $20,000 \mathrm{~km} \mathrm{~s}^{-1}$, and represents a challenging computational problem. We hope ultimately to address this problem with CASTRO [53. 59, a new adaptive mesh refinement radiationhydrodynamics code, which will allow us to follow the shock while still resolving the PNS.

\section{B. Comparison to Previous Work}

Our approach of following the collapse of a massive star's core, the formation of a natal PNS, and the subsequent off-axis motion complements previous studies that infer kicks on an excised PNS [26, 28]. By omitting the inner regions, starting the simulation $\sim 20 \mathrm{~ms}$ after bounce, and imposing a constant inner neutrino luminosity, Scheck et al. greatly reduced the problem's computational cost. They were thus able to follow the shock evolution to large distances $\left(>10^{4} \mathrm{~km}\right)$ and late times (>1 s). To approximate a physical neutron star, those authors used a contracting inner boundary motivated by radiation-hydrodynamic simulations 28]. While attractive for calculating long-term evolution, their approach requires one to infer a PNS kick through a rigid boundary of infinite inertial mass. This assumption neglects effects resulting from displacement of the PNS relative to the surrounding fluid. To compensate, in a subset of their simulations, these authors artificially add the inferred kick velocity to the gas, mimicking movement of the PNS. Our work handles all of these effects selfconsistently, providing an important check on the various approximations made in [26, 28].

Another difference between our work and that of Scheck et al. is that we implement the momentum equation in conservative form using a grid-based solution to the Poisson equation. As a result, our model conserves total momentum to better than $1 \%$ of the core's final value. Scheck et al. solve the Poisson equation using a Legendre expansion with a relativistic correction [28, 60, 61. Recently, Wongwathanarat et al. performed a three-dimensional study using the same techniques in the Scheck et al. two-dimensional studies and arrived at similar conclusions.

Given the differences in our complementary techniques, the agreement between our results and those of Scheck et al. is gratifying. Our detailed calculations of the first few hundred milliseconds including the core support the work of 26, 28, while their extended calculations indicate that a final kick magnitude of at least 400$500 \mathrm{~km} \mathrm{~s}^{-1}$ may be likely for our model. Taken together, this body of work strongly supports the case that asymmetric supernova explosions lead naturally to substantial recoil of the PNS.
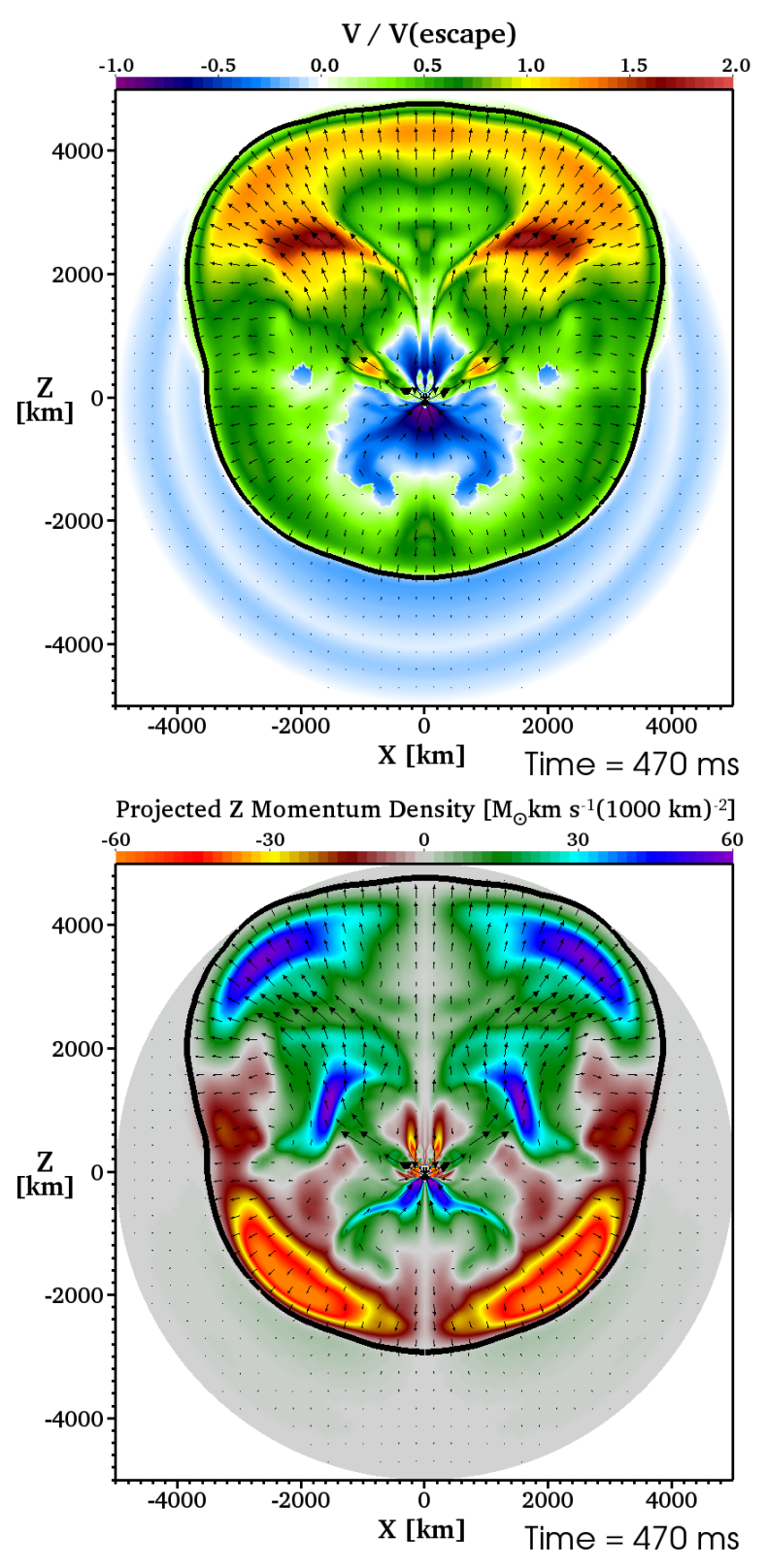

FIG. 3: Top: The ratio of the fluid velocity, $v$, to escape speed, $v_{\text {esc }}$, as a function of position $470 \mathrm{~ms}$ after bounce. Bottom: Projected $Z$-momentum density $p_{Z}$ as a function of time 470 ms after bounce. The cylindrical volume element is included, so that $\int p_{Z} \mathrm{~d} X \mathrm{~d} Z$ gives the total $Z$-momentum. We have overlaid velocity vectors and a thick black curve representing the position of the shock on both panels.

\section{CONCLUSIONS}

In this work, we have presented the first multidimensional, multi-neutrino-energy-group, radiationhydrodynamic simulation of a core-collapse supernova that results in a formation and acceleration of a nascent neutron star. The recoil of the PNS naturally arises from the asymmetric nature of the neutrino-driven explosion. 
At the end of our simulation the PNS has reached a velocity of $\sim 150 \mathrm{~km} \mathrm{~s}^{-1}$, but is still accelerating at $\sim 350$ $\mathrm{km} \mathrm{s}^{-2}$. While it is difficult to extrapolate the acceleration to later times, our PNS would need to maintain this value for only a few hundred milliseconds more to reach the peak of the observed pulsar velocity distribution. This is suggested by Fig. 3, the continued ejection of momentum in the $+Z$-direction could maintain the asymmetric matter distribution and continue to gravitationally accelerate our PNS. It should also be noted that the highest observed kicks (those upwards of $1000 \mathrm{~km}$ $\mathrm{s}^{-1}$ ) may result from the most asymmetric and energetic explosions.

Hydrodynamic recoil due to neutrino-driven, corecollapse supernovae provides a natural mechanism for accelerating neutron stars and pulsars without the need to appeal to anisotropic neutrino emission or more exotic scenarios. However, a definitive confirmation of this mechanism will require a self-consistent model of corecollapse supernova explosions. To avoid constraints imposed by axisymmetry, future work should investigate recoil and explosion anisotropies in three dimensions and compare the resulting kick velocities with observations.

\section{Acknowledgments}

J.N. and A.B. are supported by the Scientific Discovery through Advanced Computing (SciDAC) program of the DOE, under grant number DE-FG02-08ER41544, the NSF under the subaward ND201387 to the Joint Institute for Nuclear Astrophysics (JINA, NSF PHY-0822648), and the NSF PetaApps program, under award OCI0905046 via a subaward 44592 from Louisiana State University to Princeton University. Computational resources were provided by the TIGRESS high performance computer center at Princeton University, the National Energy Research Scientific Computing Center (NERSC; under contract DE-AC03-76SF00098), and on the Kraken and Ranger supercomputers, hosted at NICS and TACC via TeraGrid award TG-AST100001. This material is based upon work supported under a National Science Foundation Graduate Research Fellowship to T.D.B. C.D.O. is partially supported by the NSF under grant numbers AST-0855535 and OCI-0905046.
[1] J. E. Gunn and J. P. Ostriker, Astrophys. J. 160, 979 (1970).

[2] S. Chatterjee, W. H. T. Vlemmings, W. F. Brisken, T. J. W. Lazio, J. M. Cordes, W. M. Goss, S. E. Thorsett, E. B. Fomalont, A. G. Lyne, and M. Kramer, Astrophys. J. Lett. 630, L61 (2005), arXiv:astro-ph/0509031.

[3] J. M. Cordes and D. F. Chernoff, Astrophys. J. 505, 315 (1998), arXiv:astro-ph/9707308.

[4] W. F. Brisken, A. S. Fruchter, W. M. Goss, R. M. Herrnstein, and S. E. Thorsett, Astron. J. 126, 3090 (2003), arXiv:astro-ph/0309215.

[5] Z. Arzoumanian, D. F. Chernoff, and J. M. Cordes, Astrophys. J. 568, 289 (2002), arXiv:astro-ph/0106159.

[6] A. G. Lyne and D. R. Lorimer, Nature (London) 369, 127 (1994).

[7] B. M. S. Hansen and E. S. Phinney, Mon. Not. R. Astron. Soc. 291, 569 (1997), arXiv:astro-ph/9708071.

[8] G. Hobbs, D. R. Lorimer, A. G. Lyne, and M. Kramer, Mon. Not. R. Astron. Soc. 360, 974 (2005), arXiv:astro$\mathrm{ph} / 0504584$.

[9] W. Z. Zou, G. Hobbs, N. Wang, R. N. Manchester, X. J. $\mathrm{Wu}$, and H. X. Wang, Mon. Not. R. Astron. Soc. 362, 1189 (2005), arXiv:astro-ph/0506754.

[10] C. Faucher-Giguère and V. M. Kaspi, Astrophys. J. 643, 332 (2006), arXiv:astro-ph/0512585.

[11] H. Spruit and E. S. Phinney, Nature (London) 393, 139 (1998), arXiv:astro-ph/9803201.

[12] R. Cen, Astrophys. J. Lett. 507, L131 (1998), arXiv:astro-ph/9809022.

[13] A. M. Khokhlov, P. A. Höflich, E. S. Oran, J. C. Wheeler, L. Wang, and A. Y. Chtchelkanova, Astrophys. J. Lett. 524, L107 (1999), arXiv:astro-ph/9904419.

[14] C. D. Ott, A. Burrows, T. A. Thompson, E. Livne, and R. Walder, Astrophys. J. Suppl. 164, 130 (2006),
arXiv:astro-ph/0508462

[15] A. Burrows, L. Dessart, E. Livne, C. D. Ott, and J. Murphy, Astrophys. J. 664, 416 (2007), arXiv:astro$\mathrm{ph} / 0702539$.

[16] L. Dessart, A. Burrows, E. Livne, and C. D. Ott, Astrophys. J. 669, 585 (2007), 0705.3678.

[17] D. Lai and Y. Qian, Astrophys. J. 505, 844 (1998), arXiv:astro-ph/9802345.

[18] E. Nardi and J. I. Zuluaga, Astrophys. J. 549, 1076 (2001), arXiv:astro-ph/0006285.

[19] D. Lai, D. F. Chernoff, and J. M. Cordes, Astrophys. J. 549, 1111 (2001), arXiv:astro-ph/0007272.

[20] A. Socrates, O. Blaes, A. Hungerford, and C. L. Fryer, Astrophys. J. 632, 531 (2005), arXiv:astro-ph/0412144.

[21] A. Kusenko and G. Segrè, Phys. Rev. D 59, 061302 (1999), arXiv:astro-ph/9811144.

[22] G. Lambiase, Phys. Rev. D 71, 065005 (2005), arXiv:hep-ph/0503002.

[23] M. Barkovich, J. C. D'Olivo, and R. Montemayor, Phys. Rev. D 70, 043005 (2004), arXiv:hep-ph/0402259.

[24] G. M. Fuller, A. Kusenko, I. Mocioiu, and S. Pascoli, Phys. Rev. D 68, 103002 (2003), arXiv:astroph/0307267.

[25] A. Burrows and J. Hayes, Physical Review Letters 76, 352 (1996), arXiv:astro-ph/9511106.

[26] L. Scheck, T. Plewa, H. Janka, K. Kifonidis, and E. Müller, Physical Review Letters 92, 011103 (2004), arXiv:astro-ph/0307352.

[27] A. Burrows, L. Dessart, C. D. Ott, and E. Livne, Phys. Rep. 442, 23 (2007), arXiv:astro-ph/0612460.

[28] L. Scheck, K. Kifonidis, H. Janka, and E. Müller, Astron. Astrophys. 457, 963 (2006), arXiv:astro-ph/0601302.

[29] H. Janka and E. Mueller, Astron. Astrophys. 290, 496 (1994). 
[30] C. L. Fryer, Astrophys. J. Lett. 601, L175 (2004), arXiv:astro-ph/0312265.

[31] Y. Suwa, K. Kotake, T. Takiwaki, S. C. Whitehouse, M. Liebendoerfer, and K. Sato, ArXiv e-prints (2009), 0912.1157.

[32] K. Kjær, B. Leibundgut, C. Fransson, A. Jerkstrand, and J. Spyromilio, Astron. Astrophys. 517, A51+ (2010), 1003.5684 .

[33] E. Livne, Astrophys. J. 412, 634 (1993).

[34] E. Livne, A. Burrows, R. Walder, I. Lichtenstadt, and T. A. Thompson, Astrophys. J. 609, 277 (2004), arXiv:astro-ph/0312633.

[35] S. E. Woosley and T. A. Weaver, Astrophys. J. Supplements 101, 181 (1995).

[36] H. Shen, H. Toki, K. Oyamatsu, and K. Sumiyoshi, Nuclear Physics A 637, 435 (1998), arXiv:nucl-th/9805035.

[37] H. Shen, H. Toki, K. Oyamatsu, and K. Sumiyoshi, Progress of Theoretical Physics 100, 1013 (1998), arXiv:nucl-th/9806095.

[38] A. Burrows, E. Livne, L. Dessart, C. D. Ott, and J. Murphy, Astrophys. J. 655, 416 (2007), arXiv:astro$\mathrm{ph} / 0610175$.

[39] S. A. Colgate and R. H. White, Astrophys. J. 143, 626 (1966).

[40] J. R. Wilson, in Numerical Astrophysics, edited by J. M. Centrella, J. M. Leblanc, \& R. L. Bowers (1985), pp. $422-+$.

[41] H. A. Bethe and J. R. Wilson, Astrophys. J. 295, 14 (1985).

[42] A. Burrows, J. Hayes, and B. A. Fryxell, Astrophys. J. 450, 830 (1995), arXiv:astro-ph/9506061.

[43] A. Mezzacappa, M. Liebendörfer, O. E. Messer, W. R. Hix, F. Thielemann, and S. W. Bruenn, Physical Review Letters 86, 1935 (2001), arXiv:astro-ph/0005366.

[44] C. L. Fryer and M. S. Warren, Astrophys. J. 601, 391 (2004), arXiv:astro-ph/0309539.

[45] H. Janka and E. Mueller, Astron. Astrophys. 306, 167 (1996)

[46] H. Janka, K. Langanke, A. Marek, G. Martínez-Pinedo, and B. Müller, Phys. Rep. 442, 38 (2007), arXiv:astro$\mathrm{ph} / 0612072$.

[47] J. M. Blondin, A. Mezzacappa, and C. DeMarino, Astrophys. J. 584, 971 (2003), arXiv:astro-ph/0210634.

[48] R. Buras, M. Rampp, H. Janka, and K. Kifonidis, Astron. Astrophys. 447, 1049 (2006), arXiv:astro-ph/0507135.

[49] A. Burrows and J. Goshy, Astrophys. J. Lett. 416, L75+ (1993).

[50] M. Liebendörfer, M. Rampp, H. Janka, and A. Mezzacappa, Astrophys. J. 620, 840 (2005), arXiv:astro$\mathrm{ph} / 0310662$.

[51] A. Marek and H. Janka, Astrophys. J. 694, 664 (2009), 0708.3372 .

[52] J. W. Murphy and A. Burrows, Astrophys. J. 688, 1159 (2008), 0805.3345.

[53] J. Nordhaus, A. Burrows, A. Almgren, and J. Bell, Astrophys. J. 720, 694 (2010), 1006.3792.

[54] C. D. Ott, A. Burrows, L. Dessart, and E. Livne, Astrophys. J. 685, 1069 (2008), 0804.0239.

[55] L. Dessart, A. Burrows, E. Livne, and C. D. Ott, Astrophys. J. 645, 534 (2006), arXiv:astro-ph/0510229.

[56] T. D. Brandt, A. Burrows, and C. D. Ott, ArXiv e-prints (2010).

[57] J. M. Blondin and A. Mezzacappa, Nature (London) 445, 58 (2007), arXiv:astro-ph/0611680.

[58] R. Fernández, ArXiv e-prints (2010), 1003.1730.

[59] A. S. Almgren, V. E. Beckner, J. B. Bell, M. S. Day, L. H. Howell, C. C. Joggerst, M. J. Lijewski, A. Nonaka, M. Singer, and M. Zingale, Astrophys. J. 715, 1221 (2010), 1005.0114.

[60] M. Rampp and H. Janka, Astron. Astrophys. 396, 361 (2002), arXiv:astro-ph/0203101.

[61] A. Marek, H. Dimmelmeier, H. Janka, E. Müller, and R. Buras, Astron. Astrophys. 445, 273 (2006), arXiv:astro-ph/0502161.

[62] A. Wongwathanarat, H. Janka, and E. Mueller, ArXiv e-prints (2010), 1010.0167. 